\title{
Hyperventilationssyndrom - Das sollten Sie wissen für die Ergänzungsprüfung
}

Rico Kuhnke, Thomas Ahne

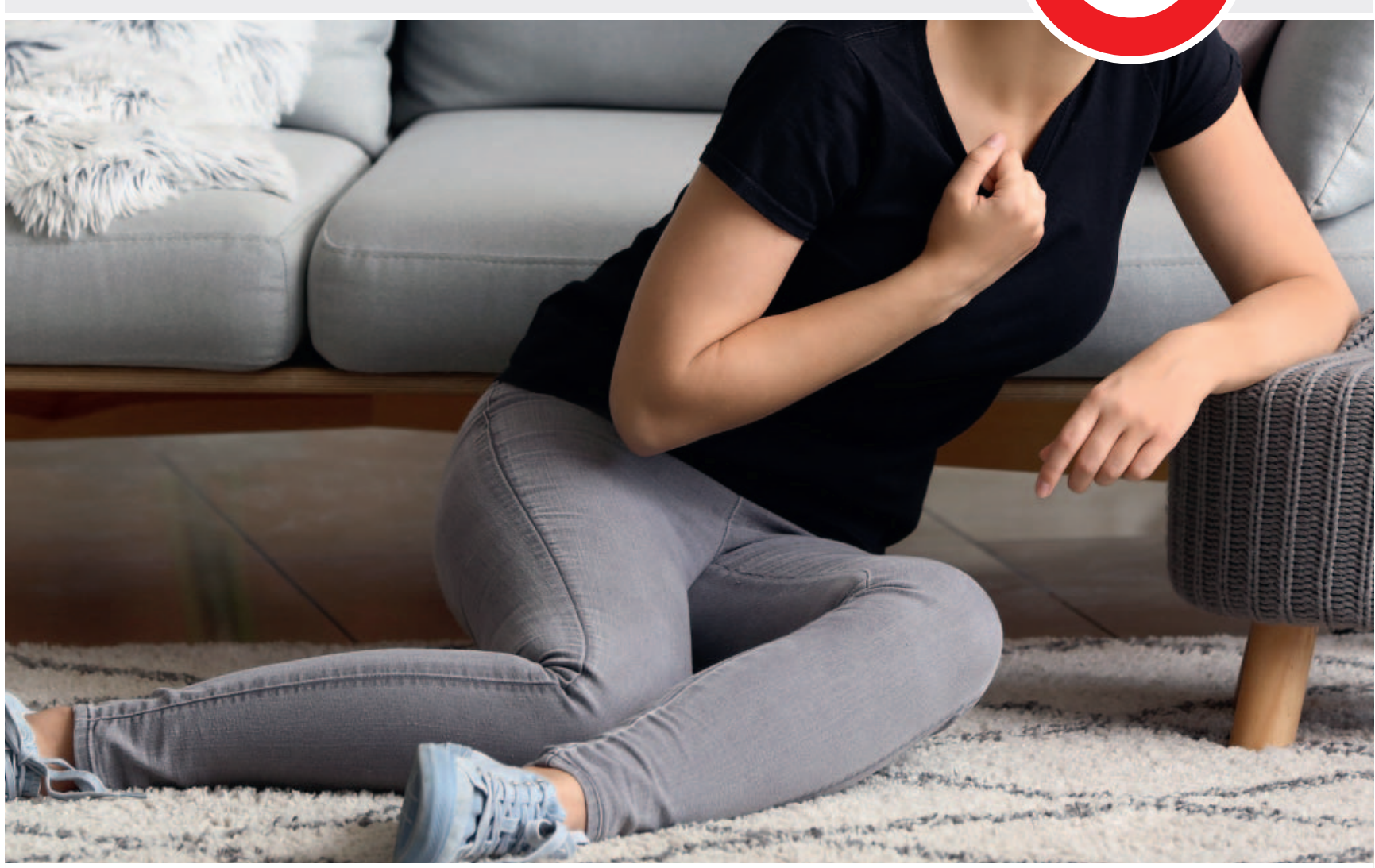

Quelle: Pixel-Shot/stock.adobe.com

retten! macht Sie fit für den Notfallsanitäter: In jeder Ausgabe arbeiten wir anhand eines Fallbeispiels einen interessanten Einsatz algorithmenkonform auf. Anhand von exemplarischen Fragen zu erweiterten Notfallmaßnahmen, Kommunikation und Rahmenbedingungen können Sie sich auf die Ergänzungsprüfung vorbereiten - egal, in welchem Bundesland Sie arbeiten.

\section{Fallbeispiel}

\section{Einsatzmeldung}

Es ist kurz vor 24 Uhr an einem sommerlichen Freitagabend, als die Besatzung des RTW zu einem Einfamilienhaus in das nahe gelegene Wohngebiet gerufen wird. Die Leitstelle meldet eine jugendliche Patientin mit akuter Atemnot.

An der Tür wartet bereits eine junge Frau und bringt das Team aufgeregt in das großzügige Wohnzimmer im Erdgeschoss. Auf dem Weg erzählt sie, sie habe gemein- sam mit ein paar „Mädels“ gefeiert und die letzte Staffel von "Game of Thrones" angeschaut. Ihre Eltern seien auf einer Städtetour in London. Der Abend sei ganz ruhig verlaufen, bis ihre Freundin plötzlich über Schwindel und zunehmende Atemnot geklagt habe. Die Symptome hätten rasch zugenommen und die Freundin habe sich nicht mehr beruhigen lassen. Daraufhin habe sie den Rettungsdienst verständigt.

\section{Situation vor Ort}

Auf einem Sessel sitzt vornübergebeugt die junge Patientin. Sie wird von ihrer besten Freundin betreut. Die 
Patientin atmet schnell und tief, die Hände scheinen verkrampft. Um sie herum stehen 4 weitere jugendliche Frauen und sind sehr aufgeregt.

Um etwas Ruhe in die Situation zu bringen, werden die jungen Frauen gebeten, in der Küche des Hauses zu warten. Die beste Freundin bleibt auf Wunsch der Patientin bei ihr. Die Anamnese gestaltet sich schwierig, da die Patientin sehr ängstlich wirkt und von Weinkrämpfen geschüttelt wird. Beiden Kollegen fällt ein intensiv aromatischer, süßlicher Geruch im Wohnzimmer auf. Da sich das Team seit langen Jahren gut kennt, genügt ein kurzer Blickkontakt, das Deuten auf die Nase und beide wissen Bescheid.

\section{Fremdanamnese}

Während sich eine Kollegin um die Patientin kümmert, erhebt der Kollege bei der besten Freundin eine Fremdanamnese. Er nutzt dazu das SAMPLER+S-Schema.

\section{S-ymptome}

Die Patientin heißt Anna und ist 16 Jahre alt. Sie habe heute zum ersten Mal geraucht. Nach ein paar tiefen Inhalationen habe sie dann plötzlich über Schwindel geklagt. Gemeinsam hätten sie versucht, Anna zu beruhigen. Diese sei aber immer panischer geworden. Immer wieder habe sie gesagt, sie bekomme keine Luft mehr. Dabei habe sie tief und schnell geatmet. Die Symptome hätten dann rasch zugenommen, Anna habe zudem über ein Taubheitsgefühl im Lippenbereich und später über Krämpfe in den Händen geklagt. Alle Bemühungen, Anna zu beruhigen, seien fehlgeschlagen. Seit einigen Minuten wimmere und weine sie nur noch.

\section{A-Ilergien}

Es sind keine Allergien bekannt.

\section{M-edikamente}

Eine regelmäßige Einnahme von Medikamenten ist der Freundin ebenfalls nicht bekannt. Auf Nachfrage gibt sie an, Anna nehme regelmäßig die Antibabypille. Die Patientin nickt bestätigend.

\section{P-atientengeschichte}

Von Vorerkrankungen weiß die Freundin nichts.

\section{L-etzte Mahlzeit, ...}

Bei dem Fest habe Anna gemeinsam mit den anderen ein paar Stücke von der selbstgemachten Pizza gegessen. Das sei ungefähr 2 Stunden her. Auch hätten sie über den Abend ein paar Gläschen Prosecco getrunken.

\section{E-reignis}

Der Kollege kommt noch einmal auf das Rauchen der Zigaretten zu sprechen. Die beste Freundin gibt sich wortkarg. Erst nach erneuter Nachfrage und dem Ansprechen auf den Geruch nach Marihuana räumt sie ein, sie hätten heute Abend gemeinsam „gekifft“. Anna habe noch nie in ihrem Leben geraucht, aber heute habe sie unbedingt probieren wollen. Es seien allerdings nur wenige Inhalationen gewesen - dann habe schon der Schwindel eingesetzt. Bei den anderen sei alles normal gewesen, nur Anna habe so stark reagiert.

\section{R-isikofaktoren}

Keine bekannt.

\section{S-chwangerschaft}

Auf die Frage nach einer Schwangerschaft schüttelt Anna den Kopf.

\section{ABCDE-Schema}

Während sich der Kollege um die Fremdanamnese kümmert, verschafft sich die Kollegin einen kurzen Überblick über die Vitalwerte. Dazu nutzt sie das ABCDE-Schema.

A-irway

Die Atemwege sind frei. Lediglich das Sprechen bereitet der Patientin Schwierigkeiten. Ein dezenter alkoholischer Foetor ist wahrnehmbar.

\section{B-reathing}

Die Atmung ist mit einer Atemfrequenz von über 40 Atemzügen pro Minute sehr schnell. Die Atemzüge sind regelmäßig und tief. Der Brustkorb hebt und senkt sich seitengleich. Beidseits ist ein unauffälliges vesikuläres Atemgeräusch zu hören. Die angelegte Pulsoxymetrie zeigt einen $\mathrm{SpO}_{2}$ von 98 \%. Die Kollegin versucht, beruhigend auf Anna einzuwirken, und leitet sie zum langsamen Atmen an.

\section{C-irculation}

Der periphere Puls an der Arteria radialis ist gut tastbar und mit einer Frequenz von 120 Schlägen pro Minute leicht tachykard. Die Rekapillarisierungzeit des Nagelbetts liegt unter 2 Sekunden. Der Blutdruck ist mit 110/70 mm Hg unauffällig.

\section{D-isability}

Anna ist wach und ansprechbar und wirkt neurologisch unauffällig. Ihre Pupillen sind isokor und mittelweit, sie reagieren prompt auf Licht. Das Blutzuckertestgerät zeigt einen BZ von $130 \mathrm{mg} / \mathrm{dl}$. 


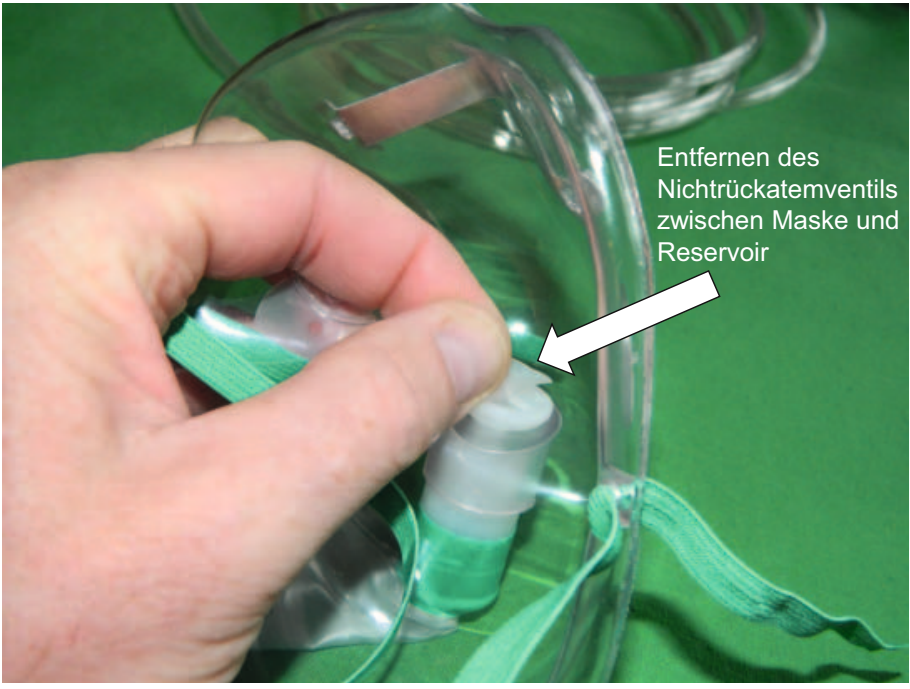

- Abb. 1 Umbau einer Venturimaske zur Hyperventilationsmaske.

\section{E-xposure}

Aufgrund der Situation wird die Patientin für eine Inspektion nicht entkleidet. Die Kollegin verschafft sich lediglich einen Überblick, achtet allerdings besonders auf Hinweise für weiteren Drogenkonsum (z. B. Einstichstellen). Es finden sich außer dem Taubheitsgefühl im Mundbereich und den verkrampften Handgelenken keine weiteren pathologischen Befunde.

\section{Merke}

Eine Übersicht über rettungsdienstliche Merkhilfen und Akronyme haben wir für Sie auf dem Plakat „Fit für den Einsatz? Das sollten Sie wissen!“ zusammengefasst. Vom SSSS-Schema über die SAMPLER-Anamnese bis zum ABCDE-Schema bietet Ihnen das Plakat eine gute Übersicht zur Vorbereitung auf Ihre Ergänzungsprüfung. Das Plakat können Sie und Ihre Kollegen auf unserer Website bestellen.

\section{0-für-10-Prinzip}

(Quelle: CRM-Leitsätze nach Rall \& Gaba in Miller's Anesthesia $7^{\text {th }}$ edition)

Das Team nimmt sich kurz Zeit, die Untersuchungsergebnisse und die weiteren Maßnahmen zu besprechen. Als Verdachtsdiagnose wird ein Hyperventilationssyndrom aufgrund einer starken psychischen Erregung angenommen. Andere Differenzialdiagnosen für eine Hyperventilation können entweder eindeutig ausgeschlossen werden (z. B. Hyperglykämie) oder sind nicht wahrscheinlich (z. B. Leberinsuffizienz).

\section{Therapie}

Da die Patientin noch nicht auf die Beruhigung angesprochen hat, entscheidet sich das Team für eine Rückatmung von Kohlendioxid. Dazu nutzt es eine Venturimaske
(Sauerstoffmaske mit Reservoir und Nichtrückatemventil). Für den Einsatz an der Patientin entfernt es den Gummipad, der das Einströmen der Ausatemluft in das Reservoir verhindern soll ( $\boldsymbol{A}$ Abb. 1). Zur Beruhigung der Patientin wird ein Sauerstoff-Flow von 2 I pro Minute eingestellt.

Auch nach 5 Minuten bleiben die Symptome bestehen. Das Team beschließt, einen venösen Zugang zu legen, und appliziert $1 \mathrm{mg}$ Dormicum (Midazolam). Kurz darauf trifft der Notarzt ein. Er entscheidet sich für eine Repetition von $1 \mathrm{mg}$ Dormicum (Midazolam). Die Symptome bessern sich zusehends und die Patientin wird in die Kinderklinik transportiert.

\section{Der Algorithmus}

Abweichend von den vorherigen Beiträgen kann das Team im beschriebenen Fall auf keinen Standardalgorithmus zurückgreifen. Da diese sich in erster Linie mit der Anwendung von erweiterten Maßnahmen durch Notfallsanitäter auseinandersetzen, gab es wohl noch keinen Grund, für das Hyperventilationssyndrom einen separaten Algorithmus zu entwerfen. Nicht selten reichen die Basismaßnahmen, wie beruhigender Zuspruch, Atemanleitung und bei Bedarf Rückatmen, nicht aus, und es wird eine medikamentöse Sedierung notwendig. Es bleibt abzuwarten, ob die Verantwortlichen in nächster Zeit die bestehenden Algorithmen um den Algorithmus zur Behandlung eines Hyperventilationssyndroms ergänzen. In Ermangelung eines Standardalgorithmus haben wir einen Musteralgorithmus entworfen, mit dem unser Team arbeitet ( $\triangleright$ Abb. 2 ).

Merke

Sollten Sie in Ihrem Rettungsdienstbereich ein abweichendes Konzept nutzen, strukturieren Sie das Fallbeispiel anhand des bei Ihnen eingesetzten Algorithmus und arbeiten Sie die Unterschiede heraus.

\section{Marihuana}

Sehr früh bemerkt das Team den aromatisch-süßlichen Geruch und vermutet, dass die jungen Frauen Marihuana geraucht haben. Die Menge, die die Patientin nach Angaben der besten Freundin inhaliert hat, ist augenscheinlich gering.

Die starke psychische Reaktion der Patientin lässt sich durch den Erstkonsum von Nikotin und THC (Tetrahydrocannabinol) erklären. Während Nikotin bei der ersten Anwendung Symptome wie Schwindel, Übelkeit oder eine Tachykardie verursachen kann, führt THC zur Veränderung des Fühlens, Denkens und der Wahrnehmung der Konsumenten. Anwender beschreiben zudem ein verändertes Körper- und Gemeinschaftserleben. In dem oben beschriebenen Fall empfindet die junge Patientin diese 
Wirkungen als bedrohlich und beängstigend und beginnt zu hyperventilieren.

\section{Karpfenmund, Ameisenkribbeln und Pfötchenstellung}

Mit anhaltender Hyperventilation beginnen die Symptome einer respiratorischen Alkalose die Patienten zusätzlich zu verängstigen. Schnell geraten sie in einen Teufelskreis aus zunehmender Angst, anhaltender Hyperventilation und sich verstärkenden Missempfindungen. Das Team schickt aus diesem Grund die anderen Gäste in die Küche nebenan und versucht, durch Entspannung der Situation und beruhigenden Zuspruch den Teufelskreis zu durchbrechen.

\section{PRAXIS/MAßNAHMEN}

Bei der Atemanleitung des Patienten ist es wichtiger, das Atemzugvolumen (AZV) zu reduzieren als die Atemfrequenz zu verlangsamen. Aufgrund des Totraumvolumens (ca. $2 \mathrm{ml} / \mathrm{kg} \mathrm{KG}$ ) kann auch bei einer vermeintlich schnellen Atmung und einem niederen AZV dem verstärkten Abatmen von $\mathrm{CO}_{2}$ entgegengewirkt werden.

\section{Differenzialdiagnose}

Das sich manifestierende Problem beim Hyperventilationssyndrom ist das verstärkte Abatmen von $\mathrm{CO}_{2}$ und damit verbunden die Entwicklung einer respiratorischen Alkalose. Vor dem Versuch der Rückatmung von $\mathrm{CO}_{2}$ müssen zwingend organische Ursachen (z. B. diabetische Ketoazidose, SHT, Vergiftungen etc.) ausgeschlossen werden.

Durch die strukturierte Anamneseerhebung mithilfe des SAMPLER+S-Schemas vermeidet das Team diagnostische Fallgruben. Da Anna das Sprechen schwerfällt, interviewt der Kollege die beste Freundin. Immer wieder versichert er sich zudem bei der Patientin, die mit Nicken und Kopfschütteln reagiert.

\section{Rückatmen}

Für das Rückatmen nutzt das Team eine umgebaute Venturimaske. Vor dem rechtlichen Hintergrund eines veränderten Medizinprodukts kann dies durchaus kritisch diskutiert werden. Steht dem Team keine spezielle Hyperventilationsmaske zur Verfügung, ist dies allemal zweckdienlicher als die Verwendung einer handelsüblichen Plastiktüte.

\section{Cave}

Zwar findet man in der Literatur immer wieder den Hinweis auf die Verwendung einer Plastiktüte zur Rückatmung, doch birgt diese das Risiko, dass der

\section{Hyperventilationssyndrom (Entwurf)}

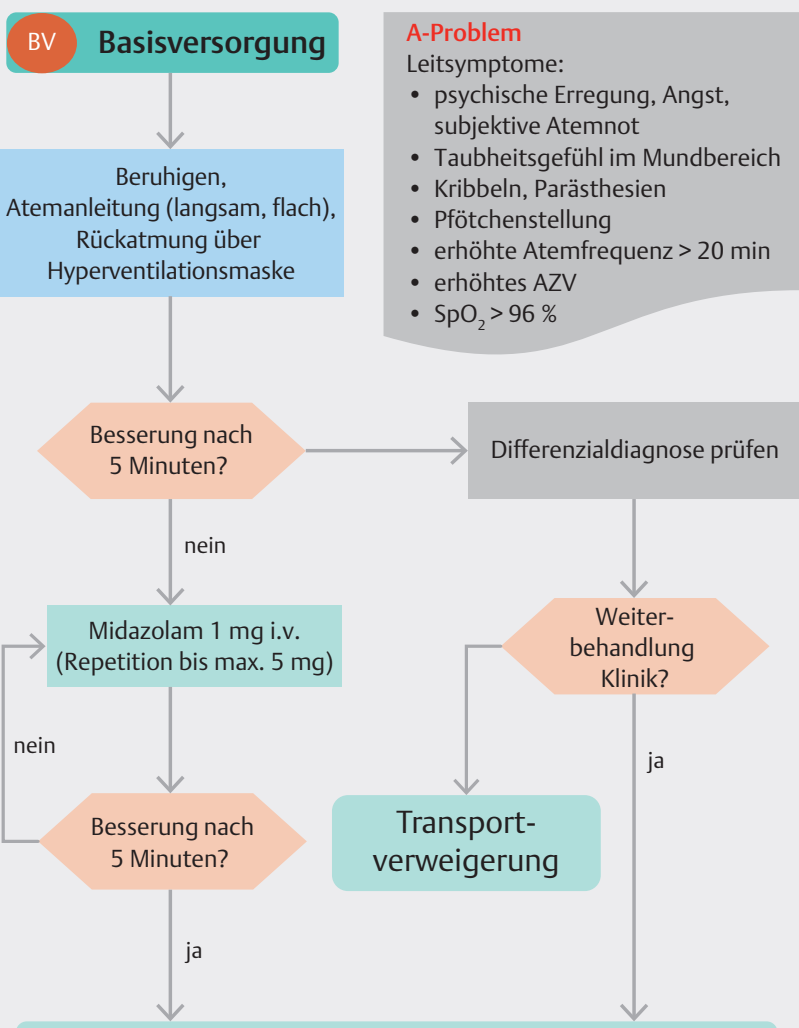

Überwachung/Transport/Übergabe/Dokumentation

Abb. 2 Musteralgorithmus Hyperventilationssyndrom.

Patient hypoxisch werden kann - insbesondere dann, wenn die Tüte zu dicht geschlossen wird und sich der Sauerstoffgehalt bei jedem Atemzug um etwa $4 \%$ reduziert. Um eine drohende Hypoxie zu vermeiden, muss darauf geachtet werden, regelmäßig Frischluft zuzuführen.

\section{PRAXIS/MAßNAHMEN}

Die Gabe von Sauerstoff bei einem Hyperventilationssyndrom ist nicht kontraindiziert. Der Patient hat zwar einen Mangel an $\mathrm{CO}_{2}$, doch die Gabe von Sauerstoff verschärft die Situation nicht weiter. Im Gegenteil kann die moderate Gabe von Sauerstoff den Patienten in seinem Gefühl einer Atemnot nachhaltig beruhigen.

Mit dem Rückatmen von $\mathrm{CO}_{2}$ werden die physiologischen Reaktionen auf das fehlende $\mathrm{CO}_{2}$ langsam reduziert. Als Faustregel gilt: Die Symptome verschwinden etwa so schnell, wie sie aufgetreten sind. 


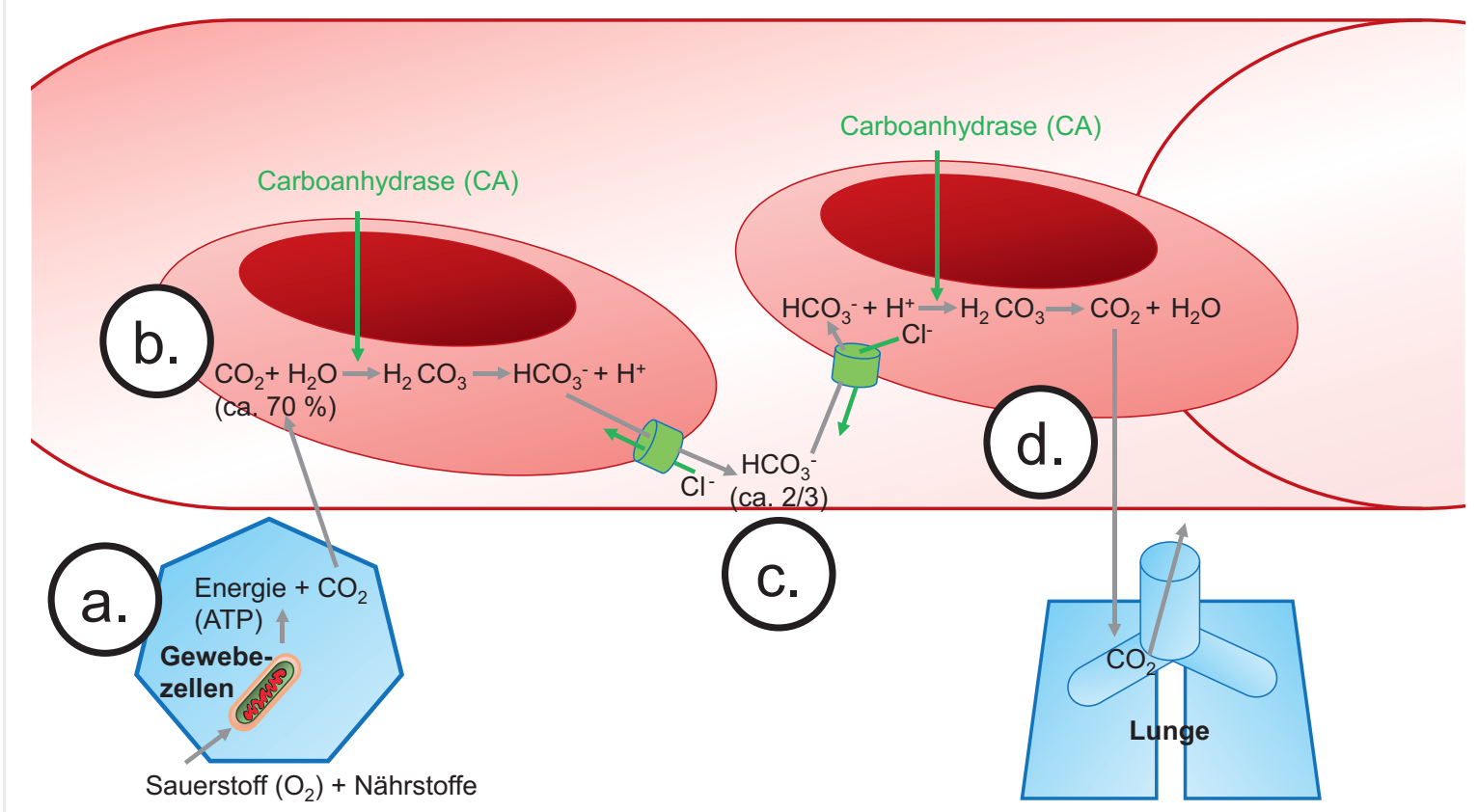

Abb. 3 Transport von $\mathrm{CO}_{2}$.

\section{Sedierung}

Da die Symptome nach 5 Minuten nicht zurückgegangen sind, beschließt das Team, die Therapie medikamentös mit Midazolam zu unterstützen. Ein Transport in die Klinik mit Überwachung ist aus diesem Grund in jedem Fall anzuraten. Im beschriebenen Fall ist die Patientin zudem minderjährig, was einen Verbleib bei den ebenfalls minderjährigen Freundinnen ausschließt.

\section{Prüfungsfragen}

\section{NOTFALLMEDIZIN}

? $\mathrm{E}$ $\mathrm{CO}_{2}$ im Blut. Beschreiben Sie die pathologischen Vorgänge bei einem Hyperventilationssyndrom ( Abb. 3).

In den Mitochondrien der Zellen werden Nährstoffe zusammen mit Sauerstoff verstoffwechselt. Neben dem Energieträger ATP und einer geringen Menge $\mathrm{H}_{2} \mathrm{O}$ entsteht als Abfallprodukt $\mathrm{CO}_{2}$. Das $\mathrm{CO}_{2}$ diffundiert in das Blutgefäßsystem und von dort in die Erythrozyten (90\%). $10 \%$ des $\mathrm{CO}_{2}$ werden im Plasma frei transportiert ( $\mathbf{A b b} \mathbf{3} \mathbf{3 a}$ ).

In Erythrozyten werden $20 \%$ des $\mathrm{CO}_{2}$ direkt an das Hämoglobin gebunden. Die verbleibenden $70 \%$ werden unterstützt durch das Enzym Carbonanhydrase (CA) über das Zwischenprodukt $\mathrm{H}_{2} \mathrm{CO}_{3}$ (Kohlensäure) in $\mathrm{HCO}_{3}{ }^{-}$(Bikarbonat) und $\mathrm{H}^{+}$(Wasserstoffion) überführt. $\mathrm{H}^{+}$wird als $\mathrm{HHb}$ am Hb (Hämoglobin) gepuffert. Rund ein Drittel des $\mathrm{HCO}_{3}{ }^{-}$bindet direkt an das $\mathrm{Hb}$, zwei Drittel werden aus den Erythrozyten ausgeschleust ( $\bullet \mathbf{A b b} \mathbf{3 b}$ ).

Das Ausschleusen von $\mathrm{HCO}_{3}{ }^{-}$geschieht über die Zellmembran des Erythrozyten im Austausch gegen $\mathrm{Cl}^{-}$(Chlorid). Dieser Vorgang wird auch als HamburgerShift bezeichnet ( $\triangleright$ Abb. $\mathbf{3 c}$ ).

Im kapillaren Endstrombereich der Lungen kehrt sich der Vorgang wieder um. Am Ende kann so das $\mathrm{CO}_{2}$ in die Lungen diffundieren und abgeatmet werden ( $\triangleright \mathbf{A b b} \mathbf{3 d}$ ).

Bei einem Hyperventilationssyndrom wird mehr $\mathrm{CO}_{2}$ abgeatmet, als durch den Stoffwechsel entstehen kann. Das zusätzliche $\mathrm{CO}_{2}$ wird aus $\mathrm{HCO}_{3}{ }^{-}$(Bikarbonat) und $\mathrm{H}^{+}$(Wasserstoffion) gewonnen. Durch die Bindung des freien $\mathrm{H}^{+}$-lons sinkt die Menge an $\mathrm{H}^{+}$im Blut, der $\mathrm{pH}$-Wert steigt (Alkalose) ( $\triangleright$ Abb. 4 ).

Anfangs wird der Verlust an $\mathrm{H}^{+}$durch Abspaltung von $\mathrm{H}^{+}$ aus den Proteinpuffern ausgeglichen. Die nun freiwerdenden Bindungsstellen werden durch freie $\mathrm{Ca}^{++}$-Ionen ersetzt. Diese beeinflussen an der Zellmembran der Muskelzellen den Einstrom von $\mathrm{Na}^{+}$und damit die Erregbarkeit der Zelle. Kommt es zu einem $\mathrm{Ca}^{++}$-Mangel, sind die Zellen leichter erregbar, und es kommt zu Muskelkontraktionen und Krämpfen. Diesem Umstand sind die typischen Symptome wie Kribbel- und Taubheitsgefühl und die Pfötchenstellung geschuldet. 


\section{Merke}

Auf den ersten Blick erscheint die Gabe von $\mathrm{Ca}^{++}$zielführend. Allerdings lindert diese nur die Symptome. Spätestens nach einer Normalisierung des Zustands und damit verbunden des Säure-Basen-Haushalts muss sich der Patient mit einem Überangebot an $\mathrm{Ca}^{++}$herumschlagen.

\section{Mögliche weitere Fragen:}

- Beschreiben Sie typische Symptome bei einem Hyperventilationssyndrom.

- An welche Differenzialdiagnosen müssen Sie denken?

- Beschreiben Sie mögliche Ursachen für eine metabolische Alkalose.

\section{KOMMUNIKATION}

? Gerade beim Hyperventilationssyndrom kommt es rasch zu einer sich aufschaukelnden Angst-Symptome-Spirale. Beschreiben Sie diesen Teufelskreis bezogen auf das obige Fallbeispiel.

Im konkreten Fall interpretiert der Körper die körperlichen Symptome als Reaktion auf den Erstkonsum von Nikotin und THC als Gefahr. Die Patientin kann die Symptome nicht deuten und wird ängstlich. Das Gefühl der Angst verursacht einen Anstieg der Atemfrequenz und der Atemtiefe. Es wird verstärkt $\mathrm{CO}_{2}$ abgeatmet und eine respiratorische Alkalose entsteht.

Anfangs sind die Symptome noch moderat (z. B. leichtes Kribbeln im Mundbereich), sie werden allerdings von der Patientin als bedrohlich wahrgenommen. Die zusätzlichen Symptome verstärken nun die Gedanken an eine latente Gefahr, die Angst nimmt weiter zu, und es kommt zu einer sich aufschaukelnden Angst-Symptome-Spirale ( $\triangleright$ Abb. 5).

\section{Mögliche weitere Fragen:}

- Beschreiben Sie mögliche Ängste der jungen Frauen, wenn die Polizei zu dem Einsatz hinzugerufen wird.

- Reflektieren Sie die Bedürfnisse der jungen Frauen.

\section{RAHMENBEDINGUNGEN}

? Alle anwesenden jungen Frauen sind unter 18 Jahren. Setzen Sie sich kritisch mit der besonderen Problematik für das Einsatzteam auseinander. Welche Möglichkeiten stehen dem Team zur Verfügung?

In Deutschland gelten Personen unter 18 Jahren als minderjährig. Minderjährige haben eingeschränkte Rechte und Pflichten und stehen unter besonderem gesetzlichem Schutz. Im konkreten Fall befindet sich kein Erziehungsberechtigter am Einsatzort. Die anwesenden Personen sind minderjährig, leicht alkoholisiert und haben Marihuana konsumiert.

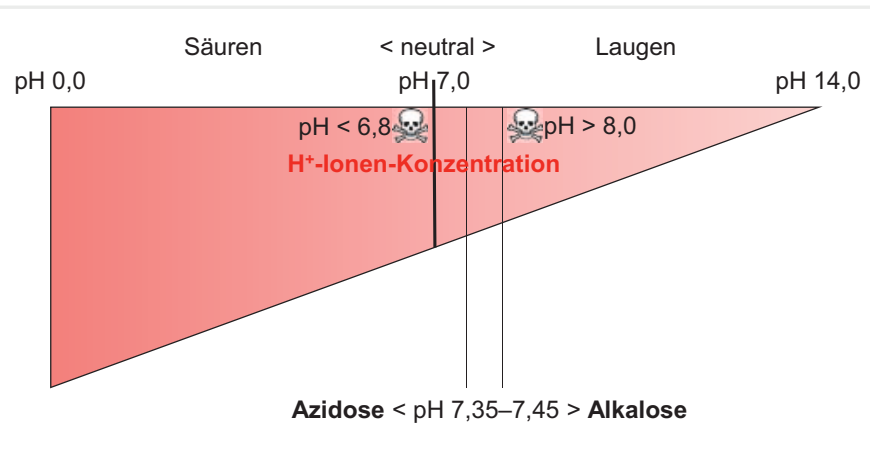

Abb. 4 pH-Wert.

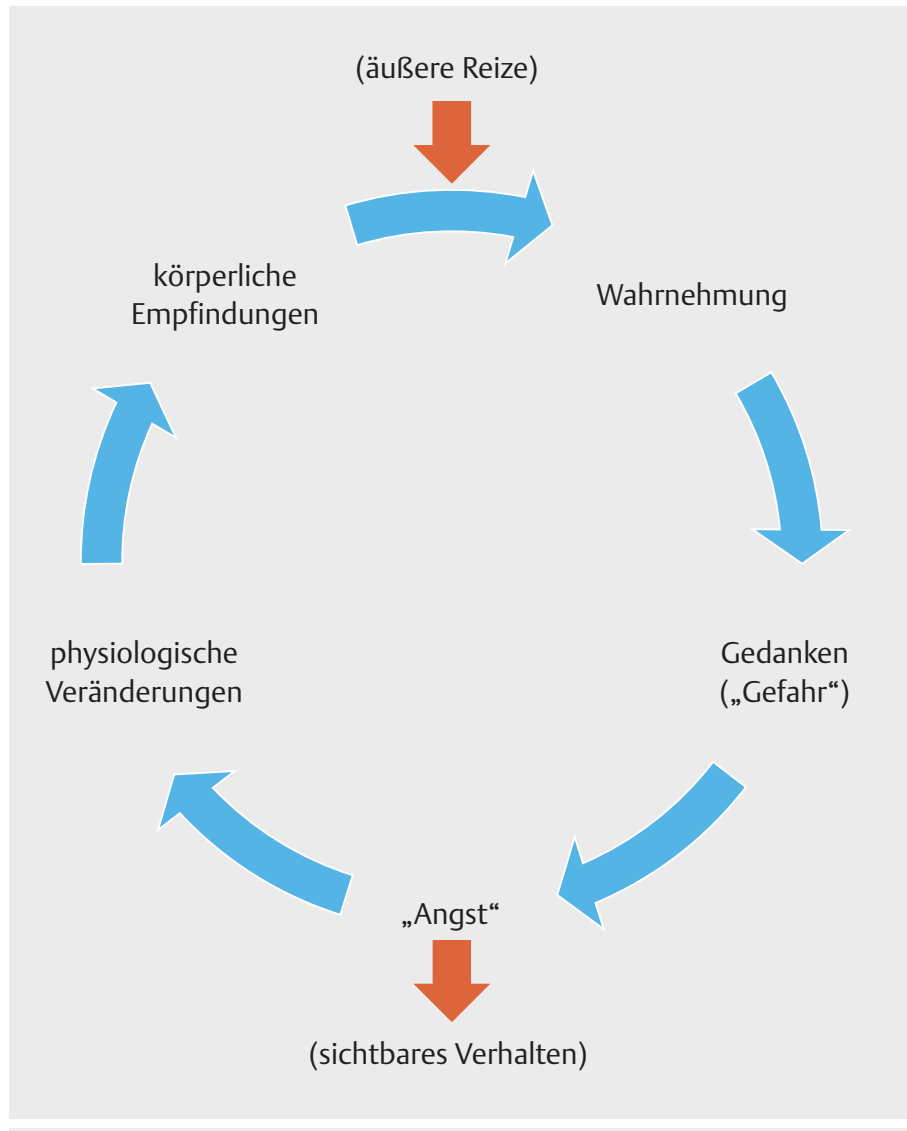

- Abb.5 Teufelskreis bei Angstanfällen nach Margraf \& Ruhmland (1996).

Ein Hinzuziehen der Polizei allein aufgrund des Marihuanas ist nicht zwingend geboten. Hier gilt es abzuwägen, welches Rechtsgut - Schweigepflicht versus Verhinderung einer Straftat - schwerer wiegt. Viel problematischer erscheint es, die Jugendlichen unbetreut zurückzulassen. Die weitere Betreuung durch mindestens einen volljährigen Erziehungsberechtigten ist unumgänglich.

Für alle Beteiligten am günstigsten wäre die Verständigung der Eltern; ggf. ist ein Elternteil bereit, die Betreuung bis zum Eintreffen der anderen zu übernehmen. Im Zweifel muss die Polizei bis zur Klärung hinzugezogen werden. 


\section{Mögliche weitere Fragen:}

- Für den oben beschriebenen Fall steht kein gesonderter Algorithmus zur Verfügung. Kann der Notfallsanitäter trotz der fehlenden SOPs Midazolam verabreichen? Begründen Sie Ihre Antwort.

- Das Team hat keine Hyperventilationsmaske zur Verfügung und nutzt daher eine leicht veränderte Venturimaske. Welche rechtliche Problematik könnte dabei bestehen?

\section{KOMMENTAR}

von Dr. med. Thomas Ahne, Facharzt für Anästhesiologie mit Zusatzbezeichnung Notfallmedizin

Ein Patient mit einem Hyperventilationssyndrom ist eine rettungsdienstliche Routinesituation. Meiner Ansicht nach kann man hier gar nicht von einer richtigen Notfallsituation sprechen, da keine Lebensbedrohung oder anhaltender gesundheitlicher Schaden befürchtet werden muss. Dennoch ist das Ereignis sehr unangenehm für den Betroffenen bzw. die Betroffene und erscheint für Laien bedrohlich, sodass ich einen Rettungsdiensteinsatz für vertretbar halte. Jedoch kann die Abarbeitung einer gemeldeten Hyperventilation auch eine große Herausforderung darstellen, nämlich wenn die Verdachtsdiagnose nicht stimmt. Aufgrund der Prägung durch das Einsatzstichwort fällt es nicht leicht, auch die Differenzialdiagnosen suffizient auszuschließen bzw. das Syndrom lediglich als Symptom einer anderen, vielleicht schwerwiegenden Erkrankung zu erkennen. Im geschilderten Fall kam es im Umfeld der Patientin auch zum Konsum von Cannabis. Dieser ist mittlerweile bei uns gesellschaftlich geduldet und daher weit verbreitet. Auf dem Vormarsch sind jedoch ebenfalls verschiedenste synthetische Rauschmittel, die oftmals auch verharmlost werden („legal highs“). Die potenziell bedrohlichen Auswirkungen des Konsums dieser Substanzen können leicht unterschätzt werden. So kann schnell ein Hyperventilationssyndrom als Erkrankungsbild missgedeutet werden, obwohl ein behandlungs- oder zumindest überwachungspflichtiges Toxidrom besteht.

So oder so muss sich das Behandlungsteam darauf konzentrieren, den Einsatz sauber abzuarbeiten, ohne sich den eigenen Bewertungs- und Wertvorstellungen zur Situation hinzugeben. Die Notwendigkeit eines erneuten Rettungsdiensteinsatzes wäre noch das kleinere Problem, aber eine durch die Missinterpretation entstehende Behandlungsverzögerung mit Patientenschaden wäre eine wahre Katastrophe.
Häufig wird in solchen Situationen schnell der Wunsch nach einer Sedierung laut, was in meinen Augen kritisch zu betrachten ist - denn dann ist auf jeden Fall eine Vorstellung im Krankenhaus zur Überwachung nach Sedation notwendig, mit den daraus resultierenden wirtschaftlichen Folgen (oftmals über $1000 €)$. Viel zielführender erscheinen mir da doch die sichere Beherrschung der Basismaßnahmen und ein empathischer Umgang mit dem Patienten bzw. der Patientin sowie den Umstehenden.

\section{Interessenkonflikt}

Die Autoren geben an, dass kein Interessenkonflikt besteht.

\section{Autorinnen/Autoren}

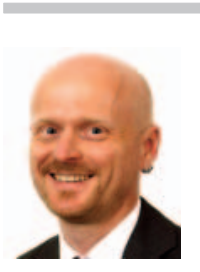

\section{Rico Kuhnke}

MA. Gesamtschulleiter der DRK-Landesschule Baden-Württemberg. Er war viele Jahre als Lehrrettungsassistent tätig und hat sein pädagogisches Masterstudium berufsbegleitend abgeschlossen. Er ist Notfallsanitäter und Mitherausgeber von retten!

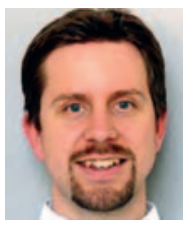

\section{Thomas Ahne}

Dr. Facharzt für Anästhesie und arbeitet derzeit als WBA Allgemeinmedizin. Er ist aktiver Notarzt und Ärztlicher Leiter Rettungsdienst des DRK Lörrach sowie Schularzt. Seine besonderen Interessensgebiete liegen im Bereich von Medical Education, Human Factors sowie der Non-Technical Skills.

Korrespondenzadresse

\section{Rico Kuhnke}

Schulleiter

Deutsches Rotes Kreuz

Landesschule Baden-Württemberg

Karl-Berner-Str. 6

72295 Pfalzgrafenweiler

r.kuhnke@drk-Is.de

\section{Bibliografie}

DOI https://doi.org/10.1055/a-1017-7201

retten 2020; 9: 88-94

(c) Georg Thieme Verlag KG, Stuttgart · New York

ISSN 2193-2387 